\title{
HOMOGENEOUS GAMES. II
}

\section{J. R. ISBELL}

Introduction. This paper describes strong simple homogeneous $n$ player games for several values of $n$ of the form $2^{k}\left(2^{l}-1\right), l>k$; specifically, for the (Mersenne) primes $2^{l}-1$ and for the first two composite values, 15, 63 (for any $k<l$ ). The problem of the existence of such a game remains open for $n=20,24,40, \cdots$.

Let us call the games fair games for short. Heuristically, a fair game of $n$ players is a rule for deciding disputed binary questions without giving any one player an advantage-for example, majority rule, if $n$ is odd. Arrow's theorem on the nonexistence of a social welfare function [2] asserts in effect that for questions which are more than binary, no fair complete rule is possible.

Precisely, a fair game on a set $N$ of players is a family of subsets of $N$, called winning sets, such that (a) every set containing a winning set is winning, (b) the complement of a winning set is not winning, (c) the complement of a nonwinning set is winning, and (d) the group of all permutations of $N$ which take winning sets to winning sets is transitive.

The problem of constructing a fair game reduces at once $[1$, Lemma 1] to the problem of constructing its group: a transitive group of permutations, every element of which has at least one odd cycle. We recall from [1] that the class of all $n$ for which a fair $n$-player game exists is closed under multiplication and contains the odd $n$ and the $n \equiv 2(\bmod 4)$, except 2 . Impossibility is known only for $n$ a power of 2 (except 1 ) and $n=12$.

1. The construction utilizes the finite projective space $P=P G(2, l-1)$ over the two-element field. Observe that $P$ has a collineation permuting its $2^{l}-1$ points cyclically [3, pp. 384-385].

Lemma. If $2^{l-1}$ is prime, 15 , or 63 , then $P G(2, l-1)$ admits a transitive collineation group $Z$ of odd order such that for any $z$ in $Z$ and any $l-1$ hyperplanes $H_{i}$ in $P$, there is $p \in P$ such that the number of points common to the orbit of $p$ under powers of $z$ and $H_{i}$, for each $i$, is odd.

Proof. Let $Z$ be a cyclic collineation group as in [3]. Specifically, for $l=4$ and $l=6$, we take $x^{4}+x+1$ and $x^{6}+x+1$ as the irreducible polynomials in Singer's construction.

Received by the editors May 25, 1959. 
If $z$ is a generator of $Z$, the orbit of any $p$ is all of $P$ and the intersection with every hyperplane is odd. If $z$ is the identity, choose $p$ common to all $H_{i}$. For primes $2^{l}-1$, there is no other case. For the case $l=4$, computation shows that the exceptional orbits are (a) lines (3 points) and (b) skew pentagons $V$ such that every plane containing two points of $V$ contains exactly three points of $V$. Each kind of orbit has odd intersection with every plane. The same thing happens for $l=6$; all exceptional orbits are unions of odd numbers of (a) lines or (b) planes. This establishes the lemma.

I do not know whether the lemma remains valid for $P G(2,7)$ or for other spaces of composite order.

2. For any $l$ satisfying the conditions of the lemma, for any $k<l$, we construct first a group $H$ of functions on $P=P G(2, l-1)$ which may be described as the direct sum of $k$ copies of the group of complements of hyperplanes. Precisely, let $S_{0}$ denote the empty set, and $S_{1}, \cdots, S_{m}\left(m=2^{l}-1\right)$ the complements of hyperplanes in $P$. The sets $S_{j}$ form a group under symmetric difference, since the symmetric difference of the complements of two hyperplanes intersecting in an $(l-3)$-subspace $T$ is the complement of the third hyperplane through $T$. Let $K$ be the direct sum of $k$ copies of $Z_{2}$, with generators $a_{1}, \cdots, a_{k}$. In the group $K^{P}$ of all functions on $P$ to $K$, let $f_{i j}(i=1, \cdots, k ; j=0, \cdots, m)$ denote the function which takes the value $a_{i}$ on $S_{j}$ and 0 on its complement. (All $f_{i 0}$ vanish.) Let $H$ be the subgroup generated by these functions. Then every element of $H$ has the form $\sum_{i=1}^{i=k} f_{i j(i)}$; for these functions include the generators $f_{i j}$ and are closed under addition. (The group is commutative, and $f_{i r}+f_{i s}=f_{i t}$ for suitable $t$.)

Next let $Q$ be an index set of $2^{k}$ elements and select a transitive action of $K$ on $Q$. (For example, let $Q$ be a product of $k$ two-element sets and let $a_{i}$ operate by changing every $i$ th coordinate.) On the product set $P \times Q$, of $2^{k} m$ elements, we define an action of $H$ by $h(p, q)=(p, h(p)(q))$. Let $Z$ be a group acting on $P$ as in the lemma, and let $Z$ act on $P \times Q$ by $z(p, q)=(z(p), q)$. Let $G$ be the least group of permutations of $P \times Q$ containing $H$ and $Z$.

Since the group of functions $H$ is invariant under collineations of $P, Z$ is contained in the normalizer of $H$ and every element of $G$ can be written (uniquely) in the form $h z$. Explicitly, $h z(p, q)$ $=(z(p), h(z(p))(q))$, and $(h z)^{s}(p, q)=\left(z^{s}(p),\left[\sum_{r=1}^{r=s} h\left(z^{r}(p)\right)\right](q)\right)$. Now the order of $z$ is an odd number, and every cycle of $z$ is odd. As for $h$, it is a sum of $k$ or fewer functions $f_{i j}$; by the lemma, there is $p$ in $P$ such that the number of points common to the orbit of $p$ under powers 
of $z$ and each $S_{j}$ is even. Let $s$ be the number of points of the orbit and $q$ any index in $Q$. For $r<s,(h z)^{r}(p, q)$ differs from $(p, q)$ in the first coordinate; but $(h z)^{8}(p, q)=(p, q)$. Thus every element of $G$ has an odd cycle. As we noted above, this implies [1] the existence of a fair game of $2^{k}\left(2^{l}-1\right)$ players.

\section{REFERENCES}

1. J. R. Isbell, Homogeneous games, Math. Student vol. 25 (1957) pp. 123-128.

2. R. D. Luce and H. Raiffa, Games and decisions: Introduction and critical survey, New York, 1957.

3. J. Singer, $A$ theorem in finite projective geometry and some applications to number theory, Trans. Amer. Math. Soc. vol. 43 (1938) pp. 377-385.

UNIVERSITY OF WASHINGTON

\section{ON INDUCED TOPOLOGIES IN QUASI-REFLEXIVE BANACH SPACES ${ }^{1}$}

\section{LARRY C. HUNTER}

1. Introduction. Let $\pi$ denote the canonical isomorphism of a Banach space $X$ into its second conjugate space $X^{* *}$. An example is given by James [4] of a space $X$ for which $X$ is separable, $X$ is not reflexive, $X$ is isomorphic to $X^{* *}$, and $X^{* *} / \pi(X)$ is one-dimensional. Civin and Yood undertook a more complete investigation of Banach spaces $X$ such that $X^{* *} / \pi(X)$ is (finite) $n$-dimensional and called such spaces quasi-reflexive Banach spaces of order $n$. If $Q$ is a subset of $X^{*}$, let $\sigma(X, Q)$ denote the least fine topology for $X$ such that all $x^{*} \in Q$ are continuous. In [1] Civin and Yood establish the following result.

THEOREM A. The following statements are equivalent:

(1) $X$ is quasi-reflexive of order $n$.

(2) There is an equivalent norm for $X$ such that $X^{*}=Q \oplus R$ where $Q$ is a total closed linear manifold such that the unit ball of $X$ is compact in $\sigma(X, Q)$ and $R$ is an n-dimensional linear manifold.

It is the purpose of this paper to study properties of the topologies $\sigma(X, Q)$, where $X^{*}=Q \oplus R, Q$ is a total closed linear manifold, and

Received by the editors December 26, 1958 and, in revised form, May 18, 1959.

1 This paper contains part of a doctoral dissertation written at the University of Oregon under the direction of Paul Civin and was supported in part by the National Science Foundation research grant NSF-G2573. 